\title{
Model-Based Testing for General Stochastic Time
}

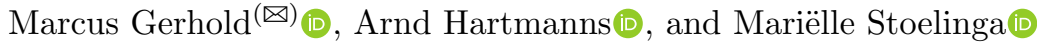 \\ University of Twente, Enschede, The Netherlands \\ \{m.gerhold,a.hartmanns\}@utwente.nl, marielle@cs.utwente.nl
}

\begin{abstract}
Many systems are inherently stochastic: they interact with unpredictable environments or use randomised algorithms. Then classical model-based testing is insufficient: it only covers functional correctness. In this paper, we present a new model-based testing framework that additionally covers the stochastic aspects in hard and soft real-time systems. Using the theory of stochastic automata for specifications, test cases and a formal notion of conformance, it provides clean mechanisms to represent underspecification, randomisation, and stochastic timing. Supporting arbitrary continuous and discrete probability distributions, the framework generalises previous work based on purely Markovian models. We cleanly define its theoretical foundations, and then outline a practical algorithm for statistical conformance testing based on the KolmogorovSmirnov test. We exemplify the framework's capabilities and tradeoffs by testing timing aspects of the Bluetooth device discovery protocol.
\end{abstract}

\section{Introduction}

Model-based testing (MBT) [29] is a technique to automatically generate, execute and evaluate test suites on black-box implementations under test (IUT). The theoretical ingredients of an MBT framework are a formal model that specifies the desired system behaviour, usually in terms of (some extension of) inputoutput transition systems; a notion of conformance that specifies when an IUT is considered a valid implementation of the model; and a precise definition of what a test case is. For the framework to be applicable in practice, we also need algorithms to derive test cases from the model, execute them on the IUT, and evaluate the results, i.e. decide conformance. They need to be sound (i.e. every implementation that fails a test case does not conform to the model), and ideally also complete (i.e. for every non-conforming implementation, there theoretically exists a failing test case). MBT is attractive due to its high degree of automation: given a model, the otherwise labour-intensive and error-prone derivation, execution and evaluation steps can be performed in a fully automatic way.

Model-based testing originally gained prominence for input-output transition systems (IOTS) using the ioco relation for input-output conformance [28]. IOTS partition the observable actions of the IUT (and thus of the model and test cases)

This work is supported by projects 3TU.BSR, NWO BEAT and NWO SUMBAT.

(C) Springer International Publishing AG, part of Springer Nature 2018

A. Dutle et al. (Eds.): NFM 2018, LNCS 10811, pp. 203-219, 2018.

https://doi.org/10.1007/978-3-319-77935-5_15 
into inputs (or stimuli) that can be provided at any time, e.g. pressing a button or receiving a network message, and outputs that are signals or activities that the environment can observe, e.g. delivering a product or sending a network message. IOTS models may include nondeterministic choices, allowing underspecification: the IUT may implement any or all of the modelled alternatives. MBT with IOTS tests for functional correctness: the IUT only exhibits behaviours allowed by the model. In the presence of nondeterminism, the IUT is allowed to use any deterministic or randomised policy to decide between the specified alternatives.

Stochastic behaviour and requirements are an important aspect of today's complex systems: network protocols extensively rely on randomised algorithms, cloud providers commit to service level agreements, probabilistic robotics [26] allows the automation of complex tasks via simple randomised strategies (as seen in e.g. vacuuming and lawn mowing robots), and we see a proliferation of probabilistic programming languages [15]. Stochastic systems must satisfy stochastic requirements. Consider the example of exponential backoff in Ethernet: an adapter that, after a collision, sometimes retransmits earlier than prescribed by the standard may not impact the overall functioning of the network, but may well gain an unfair advantage in throughput at the expense of overall network performance. In the case of cloud providers, the service level agreements are inherently stochastic when guaranteeing a certain availability (i.e. average uptime) or a certain distribution of maximum response times for different tasks. This has given rise to extensive research in stochastic model checking techniques [18]. However, in practice, testing remains the dominant technique to evaluate and certify systems outside of a limited area of highly safety-critical applications.

In this paper, we present a new MBT framework based on input-output stochastic automata (IOSA) [9], which are transition systems augmented with discrete probabilistic choices and timers whose expiration is governed by general probability distributions. By using IOSA models, we can quantitatively specify stochastic aspects of a system, in particular w.r.t. timing. We support discrete as well as continuous probability distributions, so our framework is suitable for both hard and soft real-time requirements. Since IOSA extend transition systems, nondeterminism is available for underspecification as usual. Test cases are IOSA, too, so they can naturally include waiting. We formally define the notions of stochastic ioco (sa-ioco), and of test cases as a restriction of IOSA (Sect. 3). We then outline practical algorithms for test generation and sa-ioco conformance testing (Sect.4). The latter combines per-trace functional verdicts as in standard ioco with a statistical evaluation that builds upon the Kolmogorov-Smirnov test [17]. While our theory of IOSA and sa-ioco is very general w.r.t. supported probability distributions and nondeterminism, we need to assume some restrictions to arrive at practically feasible algorithms. We finally exemplify our framework's capabilities and its inherent tradeoffs by testing timing aspects of different implementation variants of the Bluetooth device discovery protocol (Sect.5).

Related Work. Our new sa-ioco framework generalises two previous stochastic MBT approaches: the pioco framework [13] for probabilistic automata (or Markov decision processes) and marioco [14] for Markov automata (MA [12], 
which extend continuous-time Markov chains with nondeterminism). The former only supports discrete probabilistic choices and has no notion of time at all. The latter operates under the assumption that all timing is memoryless, i.e. all delays are exponentially distributed and fully characterised by means.

Early influential work had only deterministic time [3,19,21], later extended with timeouts/quiescence [4]. Probabilistic testing preorders and equivalences are well-studied $[7,10,24]$. Probabilistic bisimulation via hypothesis testing was first introduced in [20]. Our work is largely influenced by [5], which introduced a way to compare trace frequencies with collected samples. Closely related is work on stochastic finite state machines [16,23]: stochastic delays are specified similarly, but discrete probability distributions over target states are not included.

\section{Background}

Notation. $\mathbb{R}^{+}$and $\mathbb{R}_{0}^{+}$are the positive and non-negative real numbers. For a given set $\Omega$, its powerset is $\mathcal{P}(\Omega)$. A multiset is written as $\{\ldots \mid\}$. Dist $(\Omega)$ is the set of probability distributions over $\Omega$ : functions $\mu \in \Omega \rightarrow[0,1]$ s.t. $\operatorname{support}(\mu) \stackrel{\text { def }}{=}$ $\{\omega \in \Omega \mid \mu(\omega)>0\}$ is countable and $\sum_{\omega \in \operatorname{support}(\mu)} \mu(\omega)=1 . \Omega$ is measurable if it is endowed with a $\sigma$-algebra $\sigma(\Omega)$ : a collection of measurable subsets of $\Omega$. A probability measure over $\Omega$ is a function $\mu \in \sigma(\Omega) \rightarrow[0,1]$ s.t. $\mu(\Omega)=1$ and $\mu\left(\cup_{i \in I} B_{i}\right)=\sum_{i \in I} \mu\left(B_{i}\right)$ for any countable index set $I$ and pairwise disjoint measurable sets $B_{i} \subseteq \Omega$. $\operatorname{Prob}(\Omega)$ is the set of probability measures over $\Omega$. Each $\mu \in \operatorname{Dist}(\Omega)$ induces a probability measure. Let $V a l \stackrel{\text { def }}{=} V \rightarrow \mathbb{R}_{0}^{+}$be the set of valuations for an (implicit) set $V$ of (non-negative real-valued) variables. $\mathbf{0} \in \mathrm{Val}$ assigns value zero to all variables. For $X \subseteq V$ and $v \in V a l$, we write $v[X]$ for the valuation defined by $v[X](x)=0$ if $x \in X$ and $v[X](y)=v(y)$ otherwise. For $t \in \mathbb{R}_{0}^{+}, v+t$ is the defined by $(v+t)(x)=v(x)+t$ for all $x \in V$.

Stochastic automata extend Markov decision processes with stochastic clocks: real-valued variables that increase synchronously with rate 1 over time and expire some random amount of time after they have been restarted. We define SA with input/output actions along the lines of [9]:

Definition 1. An input-output stochastic automaton (IOSA) is a 6-tuple $\mathcal{I}=$ $\left\langle\right.$ Loc $\left., \mathcal{C}, \mathcal{A}, E, F, \ell_{\text {init }}\right\rangle$ where Loc is a countable set of locations, $\mathcal{C}$ is a finite set of clocks, $\mathcal{A}=\mathcal{A}^{I} \uplus \mathcal{A}^{O}$ is the finite action alphabet partitioned into inputs in $\mathcal{A}^{I}$ (marked by a ? suffix) and outputs in $\mathcal{A}^{O}$ (marked by a ! suffix), $E \in$ Loc $\rightarrow \mathcal{P}($ Edges $)$ with Edges $\stackrel{\text { def }}{=} \mathcal{P}(\mathcal{C}) \times \mathcal{A} \uplus\{\tau, \delta\} \times \operatorname{Dist}(T)$ and $T \stackrel{\text { def }}{=} \mathcal{P}(\mathcal{C}) \times$ Loc is the edge function mapping each location to a finite set of edges that in turn consist of a guard set, a label that may be the internal action $\tau$ or quiescence $\delta$, and a distribution over targets in $T$ consisting of a restart set of clocks and target locations, $F \in \mathcal{C} \rightarrow \operatorname{Prob}\left(\mathbb{R}_{0}^{+}\right)$is the delay measure function that maps each clock to a probability measure, and $\ell_{\text {init }} \in L o c$ is the initial location. $\mathcal{I}$ is input-enabled if $\forall \ell \in L o c, a \in \mathcal{A}^{I} \exists \mu:\langle\varnothing, a, \mu\rangle \in E(\ell)$. I is closed if $\mathcal{A}^{I}=\varnothing$. 
We also write $\ell \stackrel{G, a}{\longrightarrow}_{E} \mu$ for $\langle G, a, \mu\rangle \in E(\ell)$. Whenever an IOSA $\mathcal{I}_{i}$ or $\mathcal{S}_{i}$ (where index $i$ may be absent) is given in the remainder of this paper, it has the form $\left\langle L_{o c}, \mathcal{C}_{i}, \mathcal{A}_{i}, E_{i}, F_{i}, \ell_{i n i t_{i}}\right\rangle$ unless noted otherwise. Intuitively, a stochastic automaton starts its execution in the initial location with all clocks expired. An edge $\ell \stackrel{G, a}{\longrightarrow} E \mu$ may be taken only if all clocks in its guard set $G$ are expired. If any output edge (i.e. with $a \in \mathcal{A}^{O}$ ) is enabled, some edge must be taken (i.e. all outputs are urgent). When an edge is taken, (1) its action is $a,(2)$ we select a target $\left\langle R, \ell^{\prime}\right\rangle \in T$ randomly according to $\mu,(3)$ all clocks in $R$ are restarted and other expired clocks remain expired, and (4) we move to successor location $\ell^{\prime}$. There, another edge may be taken immediately or we may need to wait until some further clocks expire, and so on. When a clock $c$ is restarted, the time until it expires is chosen randomly according to the probability measure $F(c)$.

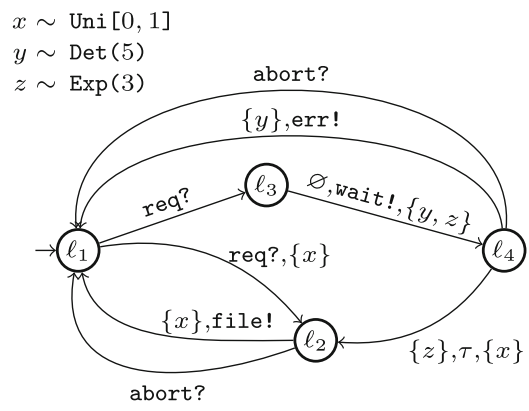

Fig. 1. File server specification.

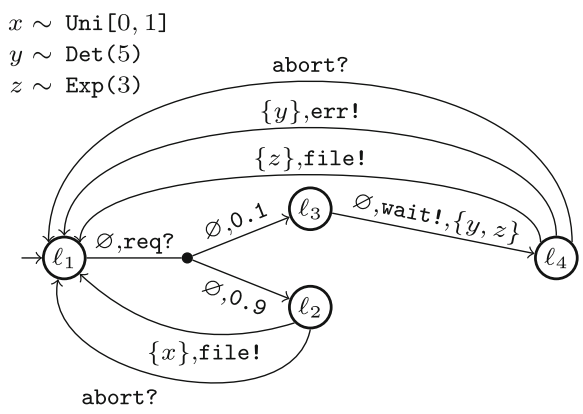

Fig. 2. File server implementation.

Example 1. Figure 1 shows an example IOSA specifying the behaviour of a file server with archival storage. We omit empty restart sets and the empty guard sets of inputs. Upon receiving a request in the initial location $\ell_{1}$, an implementation may either move to $\ell_{2}$ or $\ell_{3}$. The latter represents the case of a file in archive: the server must immediately deliver a wait! notification and then attempt to retrieve the file from the archive. Clocks $y$ and $z$ are restarted, and used to specify that retrieving the file shall take on average $\frac{1}{3}$ of a time unit, exponentially distributed, but no more than 5 time units. In location $\ell_{4}$, there is thus a race between retrieving the file and a deterministic timeout. In case of timeout, an error message (action err!) is returned; otherwise, the file can be delivered as usual from location $\ell_{2}$. Clock $x$ is used to specify the transmission time of the file: it shall be uniformly distributed between 0 and 1 time units.

In Fig. 2, we show an implementation of this specification. 1 out of 10 files randomly requires to be fetched from the archive. This is allowed by the specification: it is one particular (randomised) resolution of the nondeterministic choice, i.e. underspecification, defined in $\ell_{1}$. The implementation also manages to transmit files from archive directly while fetching them, as evidenced by the direct edge from $\ell_{4}$ back to $\ell_{1}$ labelled file !. This violates the timing prescribed by the specification, and must be detected by an MBT procedure for IOSA. 
Definition 2. Given IOSA $\mathcal{I}_{1}, \mathcal{I}_{2}$ with $\mathcal{C}_{1} \cap \mathcal{C}_{2}=\varnothing$, and $M \subseteq \mathcal{A}_{1} \times \mathcal{A}_{2}$, their parallel composition is $\mathcal{I}_{1} \| \mathcal{I}_{2} \stackrel{\text { def }}{=}\left\langle L_{o c_{1}} \times L_{o c_{2}}, \mathcal{C}_{1} \cup \mathcal{C}_{2}, \mathcal{A}_{\|}, E_{\|}, F_{1} \cup F_{2},\left\langle\ell_{\text {init }_{1}}, \ell_{\text {init }_{2}}\right\rangle\right\rangle$ where $\mathcal{A}_{\|} \stackrel{\text { def }}{=} \mathcal{A}_{\|}^{I} \uplus \mathcal{A}_{\|}^{O}$ with outputs $\mathcal{A}_{\|}^{O}=\mathcal{A}_{1}^{O} \cup \mathcal{A}_{2}^{O}$ and inputs $\mathcal{A}_{\|}^{I}=\left(\mathcal{A}_{1}^{I} \cup \mathcal{A}_{2}^{I}\right) \backslash$ $\left(\left\{a_{I} \in \mathcal{A}_{1}^{I} \mid \exists a_{O} \in \mathcal{A}_{2}^{O}:\left\langle a_{I}, a_{O}\right\rangle \in M\right\} \cup\left\{a_{I} \in \mathcal{A}_{2}^{I} \mid \exists a_{O} \in \mathcal{A}_{1}^{O}:\left\langle a_{O}, a_{I}\right\rangle \in M\right\}\right)$ and $E_{\|}$is the smallest edge function satisfying the inference rules

$$
\begin{aligned}
& \frac{\ell_{1} \stackrel{G, a}{\longrightarrow}_{E_{1}} \mu \quad a=\tau \vee \nexists a_{2} \in \mathcal{A}_{2}:\left\langle a, a_{2}\right\rangle \in M}{\left\langle\ell_{1}, \ell_{2}\right\rangle \stackrel{G, a}{\longrightarrow}_{E_{\|}}\left\{\left\langle R,\left\langle\ell_{1}^{\prime}, \ell_{2}\right\rangle\right\rangle \mapsto \mu\left(\left\langle R, \ell_{1}^{\prime}\right\rangle\right) \mid R \subseteq \mathcal{C}, \ell_{1}^{\prime} \in \operatorname{Loc}_{1}\right\}}\left(\text { indep }_{1}\right) \\
& \frac{\ell_{1} \stackrel{G_{1}, a_{1}}{\longrightarrow} E_{1} \mu_{1} \quad \ell_{2}{\stackrel{G_{2}, a_{2}}{\longrightarrow}}_{E_{2}} \mu_{2} \quad a_{1} \in \mathcal{A}_{1}^{O} \wedge\left\langle a_{1}, a_{2}\right\rangle \in M}{\left\langle\ell_{1}, \ell_{2}\right\rangle \stackrel{G_{1} \cup G_{2}, a_{1}}{\longrightarrow} E_{\|}\left\{\left\langle R_{1} \cup R_{2},\left\langle\ell_{1}^{\prime}, \ell_{2}^{\prime}\right\rangle\right\rangle \mapsto \mu\left(\left\langle R_{1}, \ell_{1}^{\prime}\right\rangle\right) \cdot \mu\left(\left\langle R_{2}, \ell_{2}^{\prime}\right\rangle\right)\right\}}\left(\text { sync }_{1}\right) \\
& \text { plus symmetric rules indep } p_{2} \text { and sync } c_{2} \text { for the corresponding steps of } \mathcal{I}_{2} \text {. }
\end{aligned}
$$

We use the convention that two actions $a_{1}$ and $a_{2}$ match, i.e. $\left\langle a_{1}, a_{2}\right\rangle \in M$, if they are the same except for the suffix (e.g. a! matches a? but not b? or a!).

Definition 3. The states of IOSA $\mathcal{I}$ are $S \stackrel{\text { def }}{=} \operatorname{Loc} \times \operatorname{Val} \times$ Val. Each $\langle\ell, v, x\rangle \in S$ consists of the current location $\ell$ and the values $v$ and expiration times $x$ of all clocks. The set of paths of $\mathcal{I}$ is Paths ${ }_{\mathcal{I}} \stackrel{\text { def }}{=} S \times\left(\mathbb{R}_{0}^{+} \times E d g e s \times \mathcal{P}(\mathcal{C}) \times S\right)^{\omega}$ where the first state is $\left\langle\ell_{i n i t}, \mathbf{0}, \mathbf{0}\right\rangle$. Paths $s_{\mathcal{I}}^{\text {fin }}$ is the set of all finite paths. For $\pi \in$ Paths ${ }_{\mathcal{I}}^{\text {fin }}$, last $(\pi)$ is its last state, and its length is the number of edges with actions $\neq \tau$.

Definition 4. A scheduler of a closed IOSA $\mathcal{I}$ is a measurable function $\mathfrak{S} \in$ $\operatorname{Sched}(\mathcal{I}) \stackrel{\text { def }}{=}$ Paths $s_{\mathcal{I}}^{\text {in }} \rightarrow \operatorname{Dist}($ Edges $\cup\{\perp\})$ such that $\mathfrak{S}(\pi)(\langle G, a, \mu\rangle)>0$ with last $(\pi)=\langle\ell, v, x\rangle$ implies $\ell \stackrel{G, a}{\longrightarrow} \mu$ and $\operatorname{Ex}(G, v+t, x)$ where $t \in \mathbb{R}_{0}^{+}$is the minimal delay for which $\nexists t^{\prime} \in\left[0, t\left[: \bigvee_{\ell} \stackrel{G^{\prime}, a^{\prime}}{\longrightarrow} \mu^{\prime} \operatorname{Ex}\left(G, v+t^{\prime}, x\right)\right.\right.$. We define $\operatorname{Ex}(G, v, x) \stackrel{\text { def }}{=} \forall c \in G: v(c) \geq x(c)$, i.e. all clocks in $G$ are expired. $\mathfrak{S}(\pi)(\perp)$ is the probability to halt. $\mathfrak{S}$ is of length $k \in \mathbb{N}$ if $\mathfrak{S}(\pi)(\perp)=1$ for all paths $\pi$ of length $\geq k$. Sched $(\mathcal{I}, k)$ is the set of all schedulers of $\mathcal{I}$ of length $k$.

A scheduler can only choose between the edges enabled at the points where any edge just became enabled in a closed IOSA. It removes all nondeterminism. The probability of each step on a path is then given by the step probability function:

Definition 5. Given $I O S A \mathcal{I}$ and $\mathfrak{S} \in \operatorname{Sched}(\mathcal{I})$, the step probability function $\operatorname{Pr}_{\mathfrak{S}} \in \operatorname{Paths}_{\mathcal{I}}^{\text {fin }} \rightarrow \operatorname{Prob}\left(\{\perp\} \cup\left(\mathbb{R}_{0}^{+} \times\right.\right.$Edges $\left.\left.\times \mathcal{P}(\mathcal{C}) \times S\right)\right)$

is defined by $\operatorname{Pr}_{\mathfrak{S}}(\pi)(\perp)=\mathfrak{S}(\pi)(\perp)$ and, for $\pi$ with last $(\pi)=\langle\ell, v, x\rangle$, $\operatorname{Pr}_{\mathfrak{S}}(\pi)\left(\left[t_{1}, t_{2}\right] \times E_{P r} \times C_{P r} \times S_{P r}\right)=$

$\mathbb{1}_{t \in\left[t_{1}, t_{2}\right]} \cdot \sum_{e=\langle G, a, \mu\rangle \in E_{P r}} \mathfrak{S}(\pi)(e) \cdot \sum_{C \in C_{P r}, \ell^{\prime} \in L o c} \mu\left(\left\langle C, \ell^{\prime}\right\rangle\right) \cdot \int_{\left\langle\ell^{\prime}, v^{\prime}, x^{\prime}\right\rangle \in S_{P r}} X_{C}^{x}\left(v^{\prime}, x^{\prime}\right)$ where $t$ is the minimal delay in $\ell$ as in Definition 4 and

$$
X_{C}^{x}\left(v^{\prime}, x^{\prime}\right)=\mathbb{1}_{v^{\prime}=(v+t)[C]} \prod_{c \in \mathcal{C}} \begin{cases}1 & \text { if } c \notin C \wedge x(c)=x^{\prime}(c) \\ 0 & \text { if } c \notin C \wedge x(c) \neq x^{\prime}(c) \\ F(c)\left(t_{2}\right)-F(c)\left(t_{1}\right) & \text { if } c \in C .\end{cases}
$$

The step probability function induces a probability measure over Paths $s_{\mathcal{I}}$. As is usual, we restrict to schedulers that let time diverge with probability 1. 
A path lets us follow exactly how an IOSA was traversed. Traces represent the knowledge of external observers. In particular, they cannot see the values of individual clocks, but only the time passed since the run started. Formally:

Definition 6. The trace of a (finite) path $\pi$ is its projection $\operatorname{tr}(\pi)$ to the delays in $\mathbb{R}_{0}^{+}$and the actions in $\mathcal{A}$. $\tau$-steps are omitted and their delays are added to that of the next visible action. The set of traces of $\mathcal{I}$ is Traces ${ }_{\mathcal{I}}$. An abstract trace in AbsTraces $\mathcal{I}$ is a sequence $\Sigma=I_{1} a_{1} I_{2} a_{2} \ldots$ with the $I_{i}$ closed intervals over $\mathbb{R}_{0}^{+}$. Finite (abstract) traces are defined analogously. Traces $s_{\mathcal{I}}^{\max }$ is the set of maximal finite traces for $\mathcal{I}$ with terminal locations. $\sigma$ represents the set of traces $\left\{t_{1} a_{1} \ldots \mid t_{i} \in I_{i}\right\}$. We identify trace $t_{1} a_{1} \ldots$ with abstract trace $\left[0, t_{1}\right] a_{1} \ldots$

We can define the trace distribution for an IOSA $\mathcal{I}$ and a scheduler as the probability measure over traces (using abstract traces to construct the corresponding $\sigma$-algebra) induced by the probability measure over paths in the usual way. The set of all finite trace distributions is $\operatorname{Tr} d(\mathcal{I})$. It induces an equivalence relation $\equiv_{T D}$ : two IOSA $\mathcal{I}$ and $\mathcal{S}$ are trace distribution equivalent, written $\mathcal{I} \equiv_{T D} \mathcal{S}$, if and only if $\operatorname{Trd}(\mathcal{I})=\operatorname{Trd}(\mathcal{S})$. A trace distribution is of length $k \in \mathbb{N}$ if it is based on a scheduler of length $k$. The set of all such trace distributions is $\operatorname{Trd}(\mathcal{I}, k)$.

\section{Stochastic Testing Theory}

We define the theoretical concepts of our MBT framework: test cases, the sa-ioco conformance relation, the evaluation of test executions, and correctness notions. The specifications $\mathcal{S}$ are IOSA as in Definition 1, and we equally assume the IUT to be an input-enabled IOSA $\mathcal{I}$ with the same alphabet as $\mathcal{S}$.

\subsection{Test Cases}

A test case describes the possible behaviour of a tester. The advantage of MBT over manual testing is that test cases can be automatically generated from the specification, and automatically executed on an implementation. In each step of the execution, the tester may either (1) send an input to the IUT, (2) wait to observe output, or (3) stop testing. A single test may provide multiple options, giving rise to multiple concrete testing sequences. It may also prescribe different reactions to different outputs. Formally, test cases for a specification $\mathcal{S}$ are IOSA whose inputs are the outputs of $\mathcal{S}$ and vice-versa. The parallel composition of either $\mathcal{S}$ or $\mathcal{I}$ with a test case thus results in a closed IOSA. By including discrete probability distributions on edges, IOSA allow making the probabilities of the three choices (input, wait, stop) explicit ${ }^{1}$. Moreover, we can use clocks for explicit waiting times in test cases. Sending input can hence be delayed, which is especially beneficial to test race conditions. A test can also react to no output being supplied, modelled by quiescence $\delta$, and check if that was expected.

\footnotetext{
${ }^{1}$ Tests are often implicitly generated probabilistically in classic ioco settings, too, without the support to make this explicit in the underlying theory. We fill this gap.
} 
Definition 7. $A$ test $\mathcal{T}$ for a specification $\mathcal{S}$ with alphabet $\mathcal{A}^{I} \uplus \mathcal{A}^{O}$ is an IOSA $\left\langle\right.$ Loc, $\left.\mathcal{C}, \mathcal{A}^{O} \uplus \mathcal{A}^{I}, E, F, \ell_{\text {init }}\right\rangle$ that has the specification's outputs as inputs and vice-versa, and that is a finite, internally deterministic, acyclic and connected tree such that for every location $\ell \in$ Loc, we either have $E(\ell)=\varnothing$ (stop testing), or $\forall \ell \stackrel{G, a}{\longrightarrow} \mu: a=\tau$ (an internal decision), or if $\exists \ell \stackrel{G, a}{\longrightarrow} \mu: a \in \mathcal{A}^{I} \cup\{\delta\}$ (we can send an input or observe quiescence) then: $\forall a_{O} \in \mathcal{A}^{O}: \exists G^{\prime}, \mu^{\prime}: \ell \stackrel{G^{\prime}, a_{O}}{\longrightarrow} \mu^{\prime}$ (all outputs can be received) and $\forall \ell \stackrel{G^{\prime}, a^{\prime}}{\longrightarrow} \mu^{\prime}: a^{\prime} \in \mathcal{A}^{O} \vee a^{\prime}=a$ (we cannot send a different input or observe quiescence in addition to an input). Whenever $\mathcal{T}$ sends an input, this input must be present in $\mathcal{S}$, too, i.e. $\forall \sigma \in \operatorname{Traces}_{\mathcal{T}}^{\text {fin }}$ with $\sigma=\sigma_{1}$ ta $\sigma_{2}$ and $a \in \mathcal{A}^{I}: \sigma_{1}$ ta $\in \operatorname{Traces}_{\mathcal{S}}^{\mathrm{fin}}$.

\subsection{Stochastic Input-Output Conformance and Annotations}

Trace distribution equivalence $\equiv_{T D}$ is the probabilistic counterpart of trace equivalence for transition systems: it shows that there is a way for the traces of two different models, e.g. the IOSA $\mathcal{S}$ and $\mathcal{I}$, to all have the same probability via some resolution of nondeterminism. However, trace equivalence or inclusion is too fine as a conformance relation for testing [27]. The ioco relation [28] for functional conformance solves the problem of fineness by allowing underspecification of functional behaviour: an implementation $\mathcal{I}$ is ioco-conforming to a specification $\mathcal{S}$ if every experiment derived from $\mathcal{S}$ executed on $\mathcal{I}$ leads to an output that was foreseen in $\mathcal{S}$. Formally:

$$
\mathcal{I} \sqsubseteq_{\text {ioco }} \mathcal{S} \Leftrightarrow \forall \sigma \in \text { Traces }_{\mathcal{S}}^{\text {fin }}: \text { out }_{\mathcal{I}}(\sigma) \subseteq \text { out }_{\mathcal{S}}(\sigma)
$$

where out $_{\mathcal{I}}(\sigma)$ is the set of outputs in $\mathcal{I}$ that is enabled after the trace $\sigma$.

Stochastic ioco. To extend ioco testing to IOSA, we need an auxiliary concept that mirrors trace prefixes stochastically: Given a trace distribution $D$ of length $k$, and a trace distribution $D^{\prime}$ of length greater or equal than $k$, we say $D$ is a prefix of $D^{\prime}$, written $D \sqsubseteq_{k} D^{\prime}$, if both assign the same probability to all abstract traces of length $k$. We can then define:

Definition 8. Let $\mathcal{S}$ and $\mathcal{I}$ be two IOSA. We say $\mathcal{I}$ is sa-ioco-conforming to $\mathcal{S}$, written $\mathcal{I} \sqsubseteq_{\text {ioco }}^{\text {sa }} \mathcal{S}$, if and only if for all tests $\mathcal{T}$ for $\mathcal{S}$ we have $\mathcal{I}\left\|\mathcal{T} \sqsubseteq_{T D} \mathcal{S}\right\| \mathcal{T}$.

Intuitively, $\mathcal{I}$ is conforming if, no matter how it resolves nondeterminism (i.e. underspecification) under a concrete test, $\mathcal{S}$ can mimic its behaviour by resolving nondeterminism for the same test, such that all traces have the same probability.

The original ioco relation takes an experiment derived from the specification and executes it on the implementation. While Definition 8 does not directly mirror this, the property implicitly carries over from the parallel composition with tests specifically designed for specifications: an input is provided to the IUT if that input is present in the specification model only.

Open schedulers. The above difference in approach between ioco and sa-ioco is due to schedulers and their resulting trace distributions being solely defined for closed systems in our work (cf. Definition 4). An alternative is to also define 
them for open systems. However, where schedulers for closed systems choose discretely between possible actions, their counterparts for open systems additionally schedule over continuous time, i.e. when an action is to be taken. This poses an additional layer of difficulty in tracing which scheduler was likely used to resolve nondeterminism, which we need to do in our a posteriori statistical analysis of the testing results (see Sect. 3.3).

Moreover, it is known $[1,6,25]$ that trace distributions of probabilistic systems under "open" schedulers are not compositional in general, i.e. $A \sqsubseteq T D B$ does not imply $A\left\|C \sqsubseteq_{T D} B\right\| C$. This would mean that, even when an implementation conforms to a specification, the execution of a probabilistic test case might tamper with the observable probabilities and yield an untrustworthy verdict. A general counterexample for the above implication is presented in [25], where however there is no requirement on input-enabledness of the composable systems. Our framework requires both implementation and test case to be inputenabled, cf. Definitions 7 and 8. The authors of [1] provide a counterexample for synchronous systems even in the presence of input-enabledness. Our framework works with input-enabled asynchronous systems; we thus believe that sa-ioco could also be defined in a way that more closely resembles the original definition of ioco by using open schedulers, but care has to be taken in defining those schedulers in the right way. We thus designed sa-ioco conservatively such that it is only based on trace semantics of closed systems, while still maintaining the possibility of underspecification as in ioco due to the way tests are used.

Annotations. To assess whether observed behaviour is functionally correct, each complete trace of a test is annotated with a verdict: all leaf locations of test cases are labelled with either pass or fail. We annotate exactly the traces that are present in the specification with the pass verdict; formally:

Definition 9. Given a test $\mathcal{T}$ for specification $\mathcal{S}$, its test annotation is the function ann $\in$ Traces $_{\mathcal{T}}^{\max } \rightarrow\{$ pass, fail $\}$ such that ann $(\sigma)=$ fail if and only if $\exists \varrho \in$

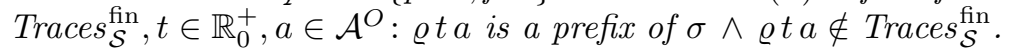

Annotations decide functional correctness only. The correctness of discrete probability choices and stochastic clocks is assessed in a separate second step.

Example 2. Figure 3 presents three test cases for the file server specification of Ex. 1. $\mathcal{T}_{1}$ uses the quiescence observation $\delta$ to assure no output is given in the initial state. $\mathcal{T}_{2}$ tests for eventual delivery of the file, which may be in archive, requiring the intermediate wait! notification, or may be sent directly. $\mathcal{T}_{3}$ utilises a clock on the abort! transition: it waits for some time (depending on what $\mathcal{T}_{3}$ specifies for $F(x)$ ) before sending the input. This highlights the ability to test for race conditions, or for the possibility of a file arrival before a specified time.

\subsection{Test Execution and Sampling}

We test stochastic systems: executing a test case $\mathcal{T}$ once is insufficient to establish sa-ioco conformance. We need many executions for an overall statistical verdict 

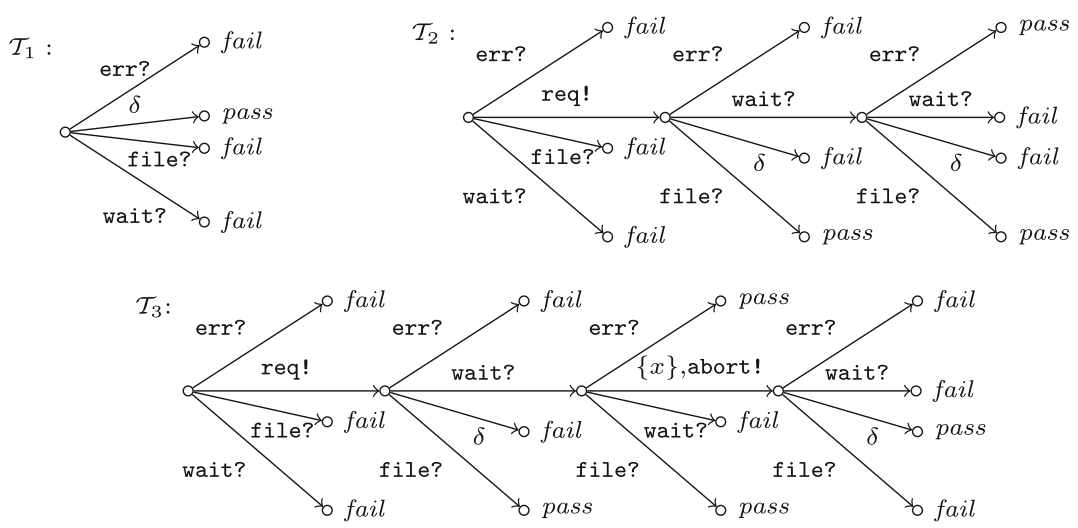

Fig. 3. Three test cases $\mathcal{T}_{1}, \mathcal{T}_{2}, \mathcal{T}_{3}$ for the file server specification.

about the stochastic behaviour in addition to the functional verdict obtained from the annotation on each execution. As establishing the functional verdict is the same as in standard ioco testing, we focus on the statistical evaluation here.

Sampling. We perform a statistical hypothesis test on the implementation based on the outcome of a push-button experiment in the sense of [22]. Before the experiment, we fix the parameters for sample length $k \in \mathbb{N}$ (the length of the individual test executions), sample width $m \in \mathbb{N}$ (how many test executions to observe), and level of significance $\alpha \in(0,1)$. The latter is a limit for the statistical error of first kind, i.e. the probability of rejecting a correct implementation. The statistical analysis is performed after collecting the sample for the chosen parameters, while functional correctness is checked during the sampling process.

Frequencies. Our goal is to determine the deviation of a sample of traces $O=$ $\left\{\sigma_{1}, \ldots, \sigma_{m}\right\}$ taken from $\mathcal{I} \| \mathcal{T}$ vs. the results expected for $\mathcal{S} \| \mathcal{T}$. If it is too large, $O$ was likely not generated by an IOSA conforming to $\mathcal{S}$ and we reject $\mathcal{I}$. If the deviation is within bounds depending on $k, m$ and $\alpha$, we have no evidence to suspect an underlying IOSA other than $\mathcal{S}$ and accept $\mathcal{I}$ as a conforming IUT. We compare the frequencies of traces in $O$ with their probabilities according to $\mathcal{S} \| \mathcal{T}$. Since $\mathcal{I}$ is a concrete implementation, the scheduler is the same for all executions, resulting in trace distribution $D$ for $\mathcal{I} \| \mathcal{T}$ and the probability of abstract trace $\Sigma$ is given directly by $D(\Sigma)$. We define $\operatorname{freq}_{O}(\Sigma) \stackrel{\text { def }}{=}|\{|\sigma \in O| \sigma \in \Sigma \mid\}| / m$, i.e. the fraction of traces in $O$ that are in $\Sigma$. $\mathcal{I}$ is rejected on statistical evidence if the distance of the two measures $D$ and $f_{r e q}$ exceeds a threshold based on $\alpha$.

Acceptable outcomes. We accept a sample $O$ if $f_{r e q}$ lies within some radius, say $r_{\alpha}$, around $D$. To minimise the error of false acceptance, we choose the smallest $r_{\alpha}$ that guarantees that the error of false rejection is not greater than $\alpha$, i.e.

$$
r_{\alpha} \stackrel{\text { def }}{=} \inf \left\{r \in \mathbb{R}^{+} \mid D\left(\text { freq }^{-1}\left(B_{r}(D)\right)\right)>1-\alpha\right\},
$$


where $B_{y}(x)$ is the closed ball centred at $x \in X$ with radius $y \in \mathbb{R}^{+}$and $X$ a metric space. The set of all measures defines a metric space together with the total variation distance of measures $\operatorname{dist}(u, v) \stackrel{\text { def }}{=} \sup _{\sigma \in\left(\mathbb{R}_{0}^{+} \times \mathcal{A}\right)^{k}}|u(\sigma)-v(\sigma)|$.

Definition 10. For $k, m \in \mathbb{N}$ and $\mathcal{I} \| \mathcal{T}$, the observations under a trace distribution $D \in \operatorname{Trd}(\mathcal{I} \| \mathcal{T}, k)$ of level of significance $\alpha \in(0,1)$ are given by the set $\operatorname{Obs}(D, \alpha, k, m)=\left\{O \in\left(\mathbb{R}_{0}^{+} \times \mathcal{A}\right)^{k \times m} \mid \operatorname{dist}\left(\right.\right.$ freq $\left.\left._{O}, D\right) \leq r_{\alpha}\right\}$.

The set of observations of $\mathcal{I} \| \mathcal{T}$ with $\alpha \in(0,1)$ is then given by the union over all trace distributions of length $k$, and is denoted $\operatorname{Obs}(\mathcal{I} \| \mathcal{T}, \alpha, k, m)$.

These sets limit the statistical error of first and second kind as follows: if a sample was generated under a trace distribution of $\mathcal{I} \| \mathcal{T}$ or a trace distribution equivalent IOSA, we accept it with probability higher than $1-\alpha$; and for all samples generated under a trace distribution by non-equivalent IOSA, the chance of erroneously accepting it is smaller than some $\beta_{m}$, where $\beta_{m}$ is unknown but minimal by construction, cf. (1). Note that $\beta_{m} \rightarrow 0$ as $m \rightarrow \infty$, i.e. the error of accepting an erroneous sample decreases as sample size increases.

\subsection{Test Evaluation and Correctness}

Definition 11. Given an IOSA $\mathcal{S}$, an annotated test case $\mathcal{T}, k$ and $m \in \mathbb{N}$, and a level of significance $\alpha \in(0,1)$, we define (1) the functional verdict as given by $v_{\text {func }} \in I O S A^{2} \rightarrow\{$ pass, fail $\}$ where $v_{\text {func }}(\mathcal{I}, \mathcal{T})=$ pass if and only if $\forall \sigma \in$ Traces $\max _{\mathcal{I} \| \mathcal{T}}^{\operatorname{Ta}} \cap$ Traces $_{\mathcal{T}}^{\max }$ : ann $(\sigma)=$ pass, and (2) the statistical verdict as given by $v_{\text {prob }} \in I O S A^{2} \rightarrow\{$ pass, fail $\}$ where $v_{\text {prob }}(\mathcal{I}, \mathcal{T})=$ pass iff $\exists D \in \operatorname{Trd}(\mathcal{S} \| \mathcal{T}, k)$ s.t.

$$
D\left(\operatorname{Obs}(\mathcal{I} \| \mathcal{T}, \alpha, k, m) \cap \operatorname{Traces}_{\mathcal{S} \mid \mathcal{T}}\right)>1-\alpha .
$$

$\mathcal{I}$ passes a test suite if $v_{\text {prob }}(\mathcal{I}, \mathcal{I})=v_{\text {func }}(\mathcal{I}, \mathcal{T})=$ pass for all annotated test cases $\mathcal{T}$ of the test suite.

The above definition connects the previous two subsections to implement a correct $\mathrm{MBT}$ procedure for the sa-ioco relation introduced in Sect. 3.2. Correctness comprises soundness and completeness (or exhaustiveness): the first means that every conforming implementation passes a test, whereas the latter implies that there is a test case to expose every erroneous (i.e. nonconforming) implementation. A test suite can only be considered correct with a guaranteed (high) probability $1-\alpha$ (as inherent in Definition 11).

Definition 12. Let $\mathcal{S}$ be a specification IOSA. Then a test case $\mathcal{T}$ is sound for $\mathcal{S}$ with respect to sa-ioco for every $\alpha \in(0,1)$ iff for every input enabled IOSA $\mathcal{I}$ we have that $\mathcal{I} \sqsubseteq_{\text {ioco }}^{\text {sa }} \mathcal{S}$ implies $v_{\text {func }}(\mathcal{I}, \mathcal{T})=v_{\text {prob }}(\mathcal{I}, \mathcal{T})=$ pass.

Completeness of a test suite is inherently a theoretical result. Infinite behaviour of the IUT, for instance caused by loops, hypothetically requires a test suite of infinite size. Moreover, there remains a possibility of accepting an erroneous implementation by chance, i.e. making an error of second kind. However, the latter is bounded from above and decreases with increasing sample size. 
Definition 13. Let $\mathcal{S}$ be a specification IOSA. Then a test suite is called complete for $\mathcal{S}$ with respect to sa-ioco for every $\alpha \in(0,1)$ iff for every input-enabled $I O S A \mathcal{I}$ we have that $\mathcal{I} \square_{\text {ioco }}^{\text {sa }} \mathcal{S}$ implies the existence of a test $\mathcal{T}$ in the test suite such that $v_{\text {func }}(\mathcal{I}, \mathcal{T})=$ fail or $v_{\text {prob }}(\mathcal{S}, \mathcal{T})=$ fail.

\section{Implementing Stochastic Testing}

The previous section laid the theoretical foundations of our new IOSA-based testing framework. Several aspects were specified very abstractly, for which we now provide practical procedures. There are already several ways to generate, annotate and execute test cases in batch or on-the-fly in the classic ioco setting [28], which can be transferred to our framework. The statistical analysis of gathered sample data in MBT, on the other hand, is largely unexplored since few frameworks include probabilities or even stochastic timing. Determining verdicts according to Definition 11 requires concrete procedures to implement the statistical tests described in Sect. 3.3 with level of significance $\alpha$. We now present practical methods to evaluate test cases in line with this theory. In particular, we need to find a scheduler for $\mathcal{S}$ that makes the observed traces $O$ most likely, and test that the stochastic timing requirements are implemented correctly.

\subsection{Goodness of Fit}

Since our models neither comprise only one specific distribution, nor one specific parameter to test for, we resort to nonparametric goodness of fit tests. Nonparametric statistical procedures allow to test hypotheses that were designed for ordinal or nominal data [17], matching our intention of (1) testing the overall distribution of trace frequencies in a sample $O=\left\{\sigma_{1}, \ldots, \sigma_{m}\right\}$, and (2) validating that the observed delays were drawn from the specified clocks and distributions. We use Pearson's $\chi^{2}$ test for (1) and multiple Kolmogorov-Smirnov tests for (2).

Pearson's $\chi^{2}$ test [17] compares empirical sample data to its expectations. It allows us to check the hypothesis that observed data indeed originates from a specified distribution. The cumulative sum of squared errors is compared to a critical value, and the hypothesis is rejected if the empiric value exceeds the threshold. We can thus check whether trace frequencies correspond to a specification under a certain trace distribution. For a finite trace $\sigma=t_{1} a_{1} t_{2} a_{2} \ldots t_{k} a_{k}$, we define its timed closure as $\bar{\sigma} \stackrel{\text { def }}{=} \mathbb{R}^{+} a_{1} \ldots \mathbb{R}^{+} a_{k}$. Applying Pearson's $\chi^{2}$ is done in general via $\chi^{2}=\sum_{i=1}^{n}\left|\operatorname{obs}_{i}-\exp _{i}\right|^{2} / \exp _{i}$, i.e. in our case

$$
\chi^{2} \stackrel{\text { def }}{=} \sum_{\bar{\sigma} \in\{\bar{\sigma} \mid \sigma \in O\}} \frac{(|\{|\bar{\varrho}| \varrho \in O \wedge \bar{\varrho}=\bar{\sigma} \mid\}| / m-D(\bar{\sigma}))^{2}}{D(\bar{\sigma})} .
$$

We need to find a $D$ that gives a high likelihood to a sample, i.e. such that $\chi^{2}<\chi_{c r i t}^{2}$, where $\chi_{\text {crit }}^{2}$ depends on $\alpha$ and the degrees of freedom. The latter is given by the number of different timed closures in $O$ minus 1 . The critical values can be calculated or found in standard tables.

Recall that a trace distribution is based on a scheduler that resolves nondeterministic choices randomly. This turns (2) into a satisfaction problem of a 
probability vector $p$ over a rational function $f(p) / g(p)$, where $f$ and $g$ are polynomials. Finding a resolution such that $\chi^{2}<\chi_{\text {crit }}^{2}$ ensures that the error of rejecting a correct IUT is at most $\alpha$. This can be done via SMT solving.

The Kolmogorov-Smirnov test. While Pearson's $\chi^{2}$ test assesses the existence of a scheduler that explains the observed trace frequencies, it does not take into account the observed delays. For this purpose, we use the non-parametric Kolmogorov-Smirnov test [17] (the KS test). It assesses whether observed data matches a hypothesised continuous probability measure. We thus restrict the practical application of our approach to IOSA where the $F(c)$ for all clocks $c$ are continuous distributions. Let $t_{1}, \ldots, t_{n}$ be the delays observed for a certain edge over multiple traces in ascending order and $F_{n}$ be the resulting step function, i.e. the right-continuous function $F_{n}$ defined by $F_{n}(t)=0$ for $t<t_{1}, F_{n}(t)=n_{i} / n$ for $t_{i} \leq t<t_{i+1}$, and $F_{n}(t)=1$ for $t \geq t_{n}$ where $n_{i}$ is the number of $t_{j}$ that are smaller or equal to $t_{i}$. Further, let $c$ be a clock with CDF $F_{c}$. Then the $n$-th KS statistic is given by

$$
K_{n} \stackrel{\text { def }}{=} \sup _{t \in \mathbb{R}_{0}^{+}}\left|F_{c}(t)-F_{n}(t)\right| .
$$

If the sample values $t_{1}, \ldots, t_{n}$ are truly drawn from the CDF $F_{x}$, then $K_{n} \rightarrow 0$ almost surely as $n \rightarrow \infty$ by the Glivenko-Cantelli theorem. Hence, for given $\alpha$ and sample size $n$, we accept the hypothesis that the $t_{i}$ were drawn from $F_{x}$ iff $K_{n} \leq K_{\text {crit }} / \sqrt{n}$, where $K_{\text {crit }}$ is a critical value given by the Kolmogorov distribution. Again, the critical values can be calculated or found in tables.

Example 3. The left-hand side of Fig. 4 shows a tiny example specification IOSA with clocks $x$ and $y$. The expiration times of both are uniformly distributed with different parameters. The right-hand side depicts a sample from this IOSA. There are two steps to assess whether the observed data is a truthful sample of the specification with a confidence of $\alpha=0.05$ : (1) find a trace distribution that minimises the $\chi^{2}$ statistic and (2) evaluate two KS tests to assess whether the observed time data is a truthful sample of $\operatorname{UNI}[0,2]$ and $\operatorname{UNI}[0,3]$, respectively.
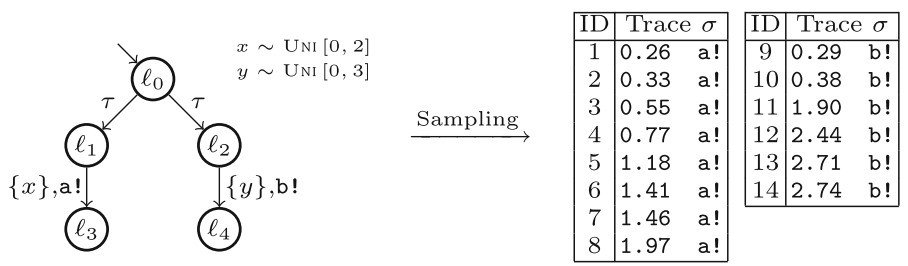

Fig. 4. Tiny example implementation IOSA and sample observation.

There are two classes of traces solely based on the action signature: ID 1-8 with a! and ID 9-14 with $\mathrm{b}$ !. Let $p$ be the probability that a scheduler assigns to taking the left branch in $\ell_{0}$ and $1-p$ that assigned to taking the right branch. Drawing a sample of size $m$, we expect $p \cdot m$ times $\mathrm{a} !$ and $(1-p) \cdot m$ times $\mathrm{b}$ !. The empirical $\chi^{2}$ value therefore calculates as 
$\chi^{2}=(8-14 \cdot p)^{2} /(14 \cdot p)+(6-14 \cdot(1-p))^{2} /(14 \cdot(1-p))$, which is minimal for $p=8 / 14$. Since it is smaller than $\chi_{\text {crit }}^{2}=3.84$, we found a scheduler that explains the observed frequencies.

Let $t_{1}=0.26, \ldots, t_{8}=1.97$ be the data associated with clock $x$ and $t_{1}^{\prime}=$ $0.29, \ldots, t_{6}^{\prime}=2.74$ be the data associated with clock $y . D_{8}=0.145$ is the maximal distance between the empirical step function of the $t_{i}$ and Uni $[0,2]$. The critical value of the Kolmogorov distribution for $n=8$ and $\alpha=0.05$ is $K_{c r i t}=0.46$. Hence, the inferred measure is sufficiently close to the specification. The KS test for $t_{i}^{\prime}$ and UNI $[0,3]$ can be performed analogously.

The acceptance of both the $\chi^{2}$ and the KS test results in the overall statistical acceptance of the implementation based on the sample data at $\alpha=0.05$.

Our intention is to provide general and universally applicable statistical tests. The KS test is conservative for general distributions, but can be made precise [8]. More specialised and thus efficient tests exist for specific distributions, e.g. the Lilliefors test [17] for Gaussian distributions, and parametric tests are generally preferred due to higher power at equal sample size. The KS test requires a comparably large sample size, and e.g. the Anderson-Darling test is an alternative.

Error propagation. A level of significance $\alpha \in(0,1)$ limits type 1 error by $\alpha$. Performing several statistical experiments inflates this probability: if one experiment is performed at $\alpha=0.05$, there is a $5 \%$ probability to incorrectly reject a true hypothesis. Performing 100 experiments, we expect to see a type 1 error 5 times. If all experiments are independent, the chance is thus $99.4 \%$. This is the family-wise error rate (FWER). There are two approaches to control the FWER: single step and sequential adjustments. The most prevalent example for the first is Bonferroni correction, while a prototype of the latter is Holm's method. Both methods aim at limiting the global type I error in the statistical testing process.

\subsection{Algorithm Outline}

The overall practical procedure to perform MBT for sa-ioco is then as follows:

1. Generate an annotated test case $\mathcal{T}$ of length $k$ for the specification IOSA $\mathcal{S}$.

2. Execute $\mathcal{T}$ on the IUT $\mathcal{I} m$ times. If the fail functional verdict is encountered in any of the $m$ test executions then fail $\mathcal{I}$ for functional reasons.

3. Calculate the number of KS tests and e.g. adjust $\alpha$ to avoid error propagation.

4. Use SMT solving to find a scheduler s.t. the $\chi^{2}$ statistic of the sample is below the critical value. If no scheduler is found, fail $\mathcal{I}$ for probabilistic reasons.

5. Group all time stamps assigned to the same clock and perform a KS test for each clock. If any of them fails, reject $\mathcal{I}$ for probabilistic reasons.

6. Otherwise, accept $\mathcal{I}$ as conforming to $\mathcal{S}$ according to $\mathcal{T}$.

Threats to validity. Step 5 has the potential to vastly grow in complexity if traces cannot be uniquely identified in the specification model. Recall Fig. 4 and assume $\mathrm{a} !=\mathrm{b} !$ : it is now infeasible to differentiate between time values belonging to the left and to the right branch. To avoid this, we have to avoid this scenario at the time of modelling, or check all possible combinations of time value assignments. 


\section{Experiments}

Bluetooth is a wireless communication protocol for low-power devices communicating over short distances. Its devices organise in small networks consisting of one master and up to seven slave devices. In this initialisation period, Bluetooth uses a frequency hopping scheme to cope with inferences. To illustrate our framework, we study the initialisation for one master and one slave device. It is inherently stochastic due to the initially random unsynchronised state of the devices. We give a high level overview and refer the reader to [11] for a detailed description and formal analysis of the protocol in a more general scenario.

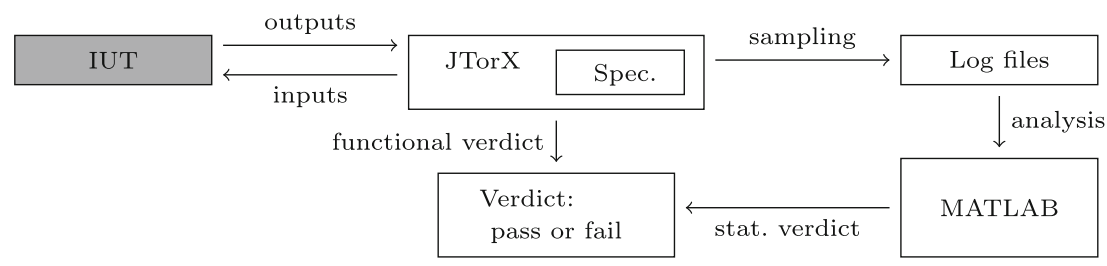

Fig. 5. Experimental setup.

Device discovery protocol. Master and slave try to connect via 32 prescribed frequencies. Both have a 28-bit clock that ticks every $312.5 \mu \mathrm{s}$. The master broadcasts on two frequencies for two consecutive ticks, followed by a two-tick listening period on the same frequencies, which are selected according to

$$
\text { freq }=\left[C L K_{16-12}+\text { off }+\left(C L K_{4-2,0}-C L K_{16-12}\right) \bmod 16\right] \bmod 32
$$

where $C L K_{i-j}$ marks the bits $i, \ldots, j$ of the clock and off $\in \mathbb{N}$ is an offset. The master switches between two tracks every $2.56 \mathrm{~s}$. When the 12 th bit of the clock changes, i.e. every $1.28 \mathrm{~s}$, a frequency is swapped between the tracks. We use off $=1$ for track 1 and off $=17$ for track 2 , i.e. the tracks initially comprise frequencies 1-16 and 17-32. The slave scans the 32 frequencies and is either in sleeping or listening state. The Bluetooth standard leaves some flexibility w.r.t. the length of the former. For our study, the slave listens for $11.25 \mathrm{~ms}$ every $0.64 \mathrm{~s}$ and sleeps for the remaining time. It picks the next frequency after $1.28 \mathrm{~s}$, enough for the master to repeatedly cycle through 16 frequencies.

Experimental setup. Our toolchain is depicted in Fig. 5. The IUT is tested onthe-fly via the MBT tool JTorX [2], which generates tests w.r.t. a transition system abstraction of our IOSA specification modelling the protocol described above. JTorX returns the functional fail verdict if unforeseen output or a timeout (quiescence) is observed at any time throughout the test process. We chose a timeout of approx. $5.2 \mathrm{~s}$ in accordance with the specification. JTorX's log files comprise the sample. We implemented the protocol and three mutants in Java 7: 
$\mathcal{M}_{1}$ Master mutant $\mathcal{M}_{1}$ never switches tracks 1 and 2, slowing the coverage of frequencies: new frequencies are only added in the swap every $1.28 \mathrm{~s}$.

$\mathcal{M}_{2}$ Master mutant $\mathcal{M}_{2}$ never swaps frequencies, only switching between tracks 1 and 2 . The expected time to connect will therefore be around $2.56 \mathrm{~s}$.

$\mathcal{S}_{1} \quad$ Slave mutant $\mathcal{S}_{1}$ has its listening period halved: it is only in a receiving state for $5.65 \mathrm{~ms}$ every $0.64 \mathrm{~s}$.

In all cases, we expect an increase in average waiting time until connection establishment. We anticipate that the increase leads to functional fail verdicts due to timeouts or to stochastic fail verdicts based on differing connection time distributions compared to the specification. We collected $m=100, m=1000$ and $m=10000$ test executions for each implementation, and used $\alpha=0.05$.

Table 1. Verdicts and Kolmogorov-Smirnov test results for Bluetooth initialisation.

\begin{tabular}{l|l|l|l|l}
\hline & Correct & \multicolumn{3}{l}{ Mutants } \\
\cline { 2 - 5 } & $\mathcal{M} \| \mathcal{S}$ & $\mathcal{M}_{1} \| \mathcal{S}$ & $\mathcal{M}_{2} \| \mathcal{S}$ & $\mathcal{M} \| \mathcal{S}_{1}$ \\
\hline$k=2$ & Accept & Reject & Accept & Accept \\
\hline$m=100$ & $D_{m}=0.065$ & - & $D_{m}=0.110$ & $D_{m}=0.065$ \\
& $D_{\text {crit }}=0.136$ & & $D_{\text {crit }}=0.136$ & $D_{\text {crit }}=0.136$ \\
\hline Timeouts & 0 & 40 & 0 & 0 \\
\hline$k=2$ & Accept & Reject & Reject & Accept \\
\hline$m=1000$ & $D_{m}=0.028$ & - & $D_{m}=0.05$ & $D_{m}=0.020$ \\
& $D_{\text {crit }}=0.045$ & & $D_{\text {crit }}=0.045$ & $D_{\text {crit }}=0.045$ \\
\hline Timeouts & 0 & 399 & 0 & 0 \\
\hline$k=2$ & Accept & Reject & Reject & Reject \\
\hline$m=10000$ & $D_{m}=0.006$ & - & $D_{m}=0.043$ & $D_{m}=0.0193$ \\
& $D_{\text {crit }}=0.019$ & & $D_{\text {crit }}=0.019$ & $D_{\text {crit }}=0.0192$ \\
\hline Timeouts & 0 & 3726 & 0 & 0 \\
\hline
\end{tabular}

Results. Table 1 shows the verdicts and the observed KS statistics $D_{m}$ alongside the corresponding critical values $D_{\text {crit }}$ for our experiments. Statistical verdict Accept was given if $D_{m}<D_{\text {crit }}$, and Reject otherwise. Note that the critical values depend on the level of significance $\alpha$ and the sample size $m$. The correct implementation was accepted in all three experiments. During the sampling of $\mathcal{M}_{1}$, we observed several timeouts leading to a functional fail verdict. It would also also have failed the KS test in all three experiments. $\mathcal{M}_{2}$ passed the test for $m=100$, but was rejected with increased sample size. $\mathcal{S}_{1}$ is the most subtle of the three mutants: it was only rejected with $m=10000$ at a narrow margin.

\section{Conclusion}

We presented an MBT setup based on stochastic automata that combines probabilistic choices and continuous stochastic time. We instantiated the theoretical 
framework with a concrete procedure using two statistical tests and explored its applicability on a communication protocol case study.

\section{References}

1. de Alfaro, L., Henzinger, T.A., Jhala, R.: Compositional methods for probabilistic systems. In: Larsen, K.G., Nielsen, M. (eds.) CONCUR 2001. LNCS, vol. 2154, pp. 351-365. Springer, Heidelberg (2001). https://doi.org/10.1007/3-540-44685-0_24

2. Belinfante, A.: JTorX: a tool for on-line model-driven test derivation and execution. In: Esparza, J., Majumdar, R. (eds.) TACAS 2010. LNCS, vol. 6015, pp. 266-270. Springer, Heidelberg (2010). https://doi.org/10.1007/978-3-642-12002-2_21

3. Bohnenkamp, H., Belinfante, A.: Timed testing with TorX. In: Fitzgerald, J., Hayes, I.J., Tarlecki, A. (eds.) FM 2005. LNCS, vol. 3582, pp. 173-188. Springer, Heidelberg (2005). https://doi.org/10.1007/11526841_13

4. Briones, L.B., Brinksma, E.: A test generation framework for quiescent real-time systems. In: Grabowski, J., Nielsen, B. (eds.) FATES 2004. LNCS, vol. 3395, pp. 64-78. Springer, Heidelberg (2005). https://doi.org/10.1007/978-3-540-31848-4_5

5. Cheung, L., Stoelinga, M., Vaandrager, F.: A testing scenario for probabilistic processes. J. ACM 54(6), 29 (2007)

6. Cheung, L., Lynch, N., Segala, R., Vaandrager, F.: Switched PIOA: parallel composition via distributed scheduling. Theor. Comput. Sci. 365(1), 83-108 (2006)

7. Cleaveland, R., Dayar, Z., Smolka, S.A., Yuen, S.: Testing preorders for probabilistic processes. Inf. Comput. 154(2), 93-148 (1999)

8. Conover, W.J.: A Kolmogorov goodness-of-fit test for discontinuous distributions. J. Am. Stat. Assoc. 67(339), 591-596 (1972)

9. D’Argenio, P.R., Lee, M.D., Monti, R.E.: Input/output stochastic automata. In: Fränzle, M., Markey, N. (eds.) FORMATS 2016. LNCS, vol. 9884, pp. 53-68. Springer, Cham (2016). https://doi.org/10.1007/978-3-319-44878-7_4

10. Deng, Y., Hennessy, M., van Glabbeek, R.J., Morgan, C.: Characterising testing preorders for finite probabilistic processes. CoRR (2008)

11. Duflot, M., Kwiatkowska, M., Norman, G., Parker, D.: A formal analysis of bluetooth device discovery. STTT 8(6), 621-632 (2006)

12. Eisentraut, C., Hermanns, H., Zhang, L.: On probabilistic automata in continuous time. In: LICS, pp. 342-351. IEEE Computer Society (2010)

13. Gerhold, M., Stoelinga, M.: Model-based testing of probabilistic systems. In: Stevens, P., Wąsowski, A. (eds.) FASE 2016. LNCS, vol. 9633, pp. 251-268. Springer, Heidelberg (2016). https://doi.org/10.1007/978-3-662-49665-7_15

14. Gerhold, M., Stoelinga, M.: Model-based testing of probabilistic systems with stochastic time. In: Gabmeyer, S., Johnsen, E.B. (eds.) TAP 2017. LNCS, vol. 10375, pp. 77-97. Springer, Cham (2017). https://doi.org/10.1007/978-3-31961467-0_5

15. Gordon, A.D., Henzinger, T.A., Nori, A.V., Rajamani, S.K.: Probabilistic programming. In: FOSE, pp. 167-181. ACM (2014)

16. Hierons, R.M., Merayo, M.G., Núñez, M.: Testing from a stochastic timed system with a fault model. J. Log. Algebr. Program. 78(2), 98-115 (2009)

17. Hollander, M., Wolfe, D.A., Chicken, E.: Nonparametric Statistical Methods. Wiley, Hoboken (2013)

18. Katoen, J.P.: The probabilistic model checking landscape. In: LICS. ACM (2016) 
19. Krichen, M., Tripakis, S.: Conformance testing for real-time systems. Form. Methods Syst. Des. 34(3), 238-304 (2009)

20. Larsen, K.G., Skou, A.: Bisimulation through probabilistic testing. ACM (1989)

21. Larsen, K.G., Mikucionis, M., Nielsen, B.: Online testing of real-time systems using UPPAAL. In: Grabowski, J., Nielsen, B. (eds.) FATES 2004. LNCS, vol. 3395, pp. 79-94. Springer, Heidelberg (2005). https://doi.org/10.1007/978-3-540-31848-4_6

22. Milner, R. (ed.): A Calculus of Communicating Systems. LNCS, vol. 92. Springer, Heidelberg (1980). https://doi.org/10.1007/3-540-10235-3

23. Núñez, M., Rodríguez, I.: Towards testing stochastic timed systems. In: König, H., Heiner, M., Wolisz, A. (eds.) FORTE 2003. LNCS, vol. 2767, pp. 335-350. Springer, Heidelberg (2003). https://doi.org/10.1007/978-3-540-39979-7_22

24. Segala, R.: Modeling and verification of randomized distributed real-time systems. Ph.D. thesis, Cambridge, MA, USA (1995)

25. Stoelinga, M.: Alea jacta est: verification of probabilistic, real-time and parametric systems. Ph.D. thesis, Radboud University of Nijmegen (2002)

26. Thrun, S., Burgard, W., Fox, D.: Probabilistic Robotics. MIT press, Cambridge (2005)

27. Tretmans, J.: Conformance testing with labelled transition systems: implementation relations and test generation. Comput. Netw. ISDN Syst. 29(1), 49-79 (1996)

28. Tretmans, J.: Model based testing with labelled transition systems. In: Hierons, R.M., Bowen, J.P., Harman, M. (eds.) Formal Methods and Testing. LNCS, vol. 4949, pp. 1-38. Springer, Heidelberg (2008). https://doi.org/10.1007/978-3-54078917-8_1

29. Utting, M., Pretschner, A., Legeard, B.: A taxonomy of model-based testing approaches. Softw. Test. Verif. Reliab. 22(5), 297-312 (2012) 